La

Révolution

française

\section{La Révolution française}

Cahiers de l'Institut d'histoire de la Révolution française

$2 \mid 2012$

La presse

\title{
Encyclopédisme et distribution des savoirs. Le cas du Magasin encyclopédique. 1795-1816
}

Encyclopedism and Distribution of Knowledge. The Magasin encyclopédique. 1795-1816.

Pierre-Yves Lacour

\section{OpenEdition}

\section{Journals}

Édition électronique

URL : http://journals.openedition.org//rf/588

DOI : $10.4000 /$ Irf.588

ISSN : 2105-2557

Éditeur

IHMC - Institut d'histoire moderne et contemporaine (UMR 8066)

Référence électronique

Pierre-Yves Lacour, «Encyclopédisme et distribution des savoirs. Le cas du Magasin encyclopédique. 1795-1816 », La Révolution française [En ligne], 2 | 2012, mis en ligne le 15 septembre 2012, consulté le 19 avril 2019. URL : http://journals.openedition.org/Irf/588 ; DOI : 10.4000/Irf.588

Ce document a été généré automatiquement le 19 avril 2019

(c) La Révolution française 


\section{Encyclopédisme et distribution des savoirs. Le cas du Magasin encyclopédique. 1795-1816}

Encyclopedism and Distribution of Knowledge. The Magasin encyclopédique. 1795-1816.

Pierre-Yves Lacour

Entre 1795 et 1816, Aubin-Louis Millin (1759-1818) publie le Magasin encyclopédique. La personnalité du publiciste Millin est essentielle pour comprendre la nature et le fonctionnement $\mathrm{du}$ périodique qu'il dirige ${ }^{1}$. Figure exemplaire du polygraphe des Lumières, il est à la fois naturaliste et antiquaire, cette seconde passion l'emportant progressivement sur la première au cours de la décennie révolutionnaire. Comme secrétaire de la Société d'Histoire naturelle de Paris, il pétitionne en faveur d'un voyage à la recherche de La Pérouse, donne des Instructions aux naturalistes de l'expédition d'Entrecasteaux et traduit des ouvrages de Linné et Fabricius. Conservateur du département des médailles et des antiquités de la Bib liothèque nationale depuis 1795, il y étudie les « mœurs et usages chez les Anciens par les monumens » et y donne des cours de bibliographie et d'archéologie. Il entame aussi la publication des Antiquités nationales avant de devenir membre de la classe d'Histoire et littérature ancienne de l'Institut. Le rédacteur du Magasin est aussi en correspondance avec de très nombreux antiquaires et naturalistes européens de même qu'il prend part à une foule de sociétés savantes. Une fois par décade, il tient salon dans son appartement de fonction où la vieille mère du conservateur sert le thé à toute l'Europe savante et mondaine ${ }^{2}$. On y croise aussi régulièrement une cinquantaine de personnes: les conservateurs de la Bibliothèque nationale, des professeurs des Écoles centrales, des députés, des hellénistes, des chimistes, des naturalistes et, parmi tous ceux-ci, de nombreux étrangers, principalement des Allemands ${ }^{3}$. Le fameux « thé littéraire » chez Millin, qui est attesté à partir de 1797 et prend fin en 1811 après un long déclin, est l'un des principaux espaces de la sociabilité post-révolutionnaire. La publication du Magasin repose largement sur ces trois espaces savants, la correspondance privée, le salon particulier et la bibliothèque publique : par ses 
nombreuses relations épistolaires, Millin se tient informé des derniers développements en histoire naturelle et dans l'étude des antiquités; de par sa position à la Bibliothèque nationale, il reçoit toutes les nouvelles parutions savantes qu'il propose aux visiteurs de son salon; elles y sont commentées par «des groupes où l'on [joue] les juges avec gravité » selon le voyageur Rist, le salon formant alors l'antichambre du Magasin ${ }^{4}$. En retour, la fonction de publiciste renforce la position de Millin dans le champ naturaliste et antiquaire.

2 Avec la Décade philosophique, le Magasin est le principal périodique encyclopédique de la période révolutionnaire et impériale. Pourtant, à la différence de sa consœur, il n'a donné lieu qu'à peu de travaux universitaires à l'exception notable d'un volume de contributions rassemblé par Geneviève Espagne et Bénédicte Savoy sous le titre Aubin-Louis Millin et l'Allemagne $e^{5}$. Entre décembre 1792 et mars 1793, 52 numéros du Magasin sont publiés. Le périodique se décrit alors comme « uniquement consacré à entretenir entre les savans, les littérateurs \& les artistes, la communication nécessaire aux travaux dont ils s'occupent $»^{6}$. La publication de ces numéros réunis en un seul volume s'interrompt brusquement lors de l'emprisonnement de Millin entre septembre 1793 et août 1794. Dès 1795, Millin reprend la parution du Magasin. Il est d'abord aidé par François-Joseph Noël et Israël Warens puis, dès 1796, il dirige à nouveau seul la publication jusqu'à la disparition du périodique vingt années plus tard ${ }^{7}$. De 1795 à 1816, six volumes sont publiés par année, chacun contenant six numéros, soit un par décade. Au total, la série du Magasin encyclopédique se compose de 122 volumes de 475 à 575 pages in-octavo. Selon une formule partagée par de nombreux périodiques savants, chaque numéro du Magasin renferme des articles originaux, des lettres de correspondants, des extraits d'ouvrages, des rapports à l'Institut national, des comptes-rendus de livres et des annonces diverses ${ }^{8}$. Important aux yeux de l'historien de la culture, le Magasin ne connaît qu'un succès très relatif pendant la vingtaine d'années de son existence. En 1803, dans un rapport au premier Consul, Roederer parle pour le Magasin d'environ une centaine de souscripteurs en province; Eugène Hattin et Marc Régaldo pensent que le nombre total des abonnés au niveau national doit être compris entre 150 et $200^{\circ}$. Vers 1804, John Pinkerton estime que le tirage du Magasin encyclopédique comme celui de la Décade philosophique, ne doit pas excéder les 300 exemplaires $^{10}$. En 1812, dans une lettre à Silvestre de Sacy, Christian Friedrich Schnurrer évoque un tirage à 500 exemplaires $^{11}$. On dira environ 300 exemplaires.

Dans le Magasin de Millin comme dans l'Encyclopédie de Diderot et d'Alembert, le projet encyclopédique désigne simultanément deux choses. D’une part, il signale la réunion des sciences et des arts, comme l'indique le sous-titre du Magasin qui annonce un «Journal des Sciences, des Lettres et des Arts ». En juillet 1795, on lit dans la revue allemande Minerva que le Magasin « dont sont déjà sortis six numéros [...] recouvre, d'après son plan, toutes les sciences et tous les arts. La politique seule a été très sagement non pas expressément exclue, mais, à ce qu'il semble, supprimée pour ne pas faire d'une revue destinée à l'instruction générale le dépôt d'opinions privées choquantes »" ${ }^{12}$. En 1796, dans un avertissement, on lit encore qu'il s'agit d'un "journal purement littéraire», c'est-àdire explicitement dépourvu de considérations politiques, et qu'il doit pouvoir se substituer à une foule d'anciens journaux généralistes et spécialisés alors disparus ${ }^{13}$. D'autre part, la notion d'encyclopédisme implique l'articulation des différents domaines de savoir. Dans un article de la Décade de décembre 1794, Millin préconise une méthode analytique pour ordonner les livres des bibliothèques : il écrit que «l'ordre alphabétique 
est toujours le plus mauvais, parce qu'il ne case rien dans l'entendement » et demande une méthode, renvoyant explicitement au "Système figuré des connaissances" de l' Encyclopédie et prenant comme modèle les classifications naturalistes ${ }^{14}$. L'encyclopédisme renvoie donc tout à la fois à la réunion des sciences et des arts selon une formule souvent employée et à la recherche d'un ordre, c'est-à-dire d'une articulation des savoirs. Cette tentative d'ordonner les connaissances apparaît dans les «tables des matières » du Magasin. Dans Aubin-Louis Millin et l'Allemagne, plusieurs études portent sur des domaines de savoir particuliers comme l'orientalisme, l'histoire ou la philologie. La démarche des auteurs y est ouvertement généalogique. Partant de l'acception actuelle d'une discipline pour dresser la cartographie de sa dispersion dans les tables du Magasin encyclopédique, ils proposent la figure de savoirs en archipels : le champ de la philologie est éclaté dans une multitude de rubriques; celui de l'histoire partagé entre les rubriques Histoire, Archéologie, Voyages ou Philosophie; l'orientalisme est représenté dans diverses rubriques Littérature, Voyages ou Nouvelles littéraires ${ }^{15}$. Nous adopterons la démarche inverse en partant de l'ordonnancement des tables telles qu'elles sont présentées à la fin de chacun des 122 volumes. Nous nous demanderons quelle est la place des différents savoirs dans les tables du Magasin encyclopédique, la "place ", c'est-à-dire aussi bien la position de chaque rubrique dans une configuration générale que le poids relatif de chaque domaine de savoir dans l'ensemble des notices.

\section{L'enchaînement des savoirs}

Dans les tables du Magasin, les savoirs ne sont pas hiérarchisés sous la forme d'un arbre des connaissances ou d'un système figuré qui étendrait loin ses ramifications. Seule exception, la zoologie dont Millin désigne les embranchements par un jeu typographique entre grandes et petites capitales pour désigner rubriques et sous-rubriques. Chacune des tables des 122 volumes se présente de la même manière : le nom de la rubrique est imprimé en grandes capitales et centré sur la page ; les titres des articles qui suivent sont en caractère minuscule et les lignes justifiées. La ronde des savoirs semble alors se déplier sous la forme d'une liste souvent encadrée par deux catégories généralistes, «sciences et arts » et « mélanges ». 
Fig. 1 : Première page de la table des matières du deuxième volume de la deuxième année du Magasin encyclopédique.

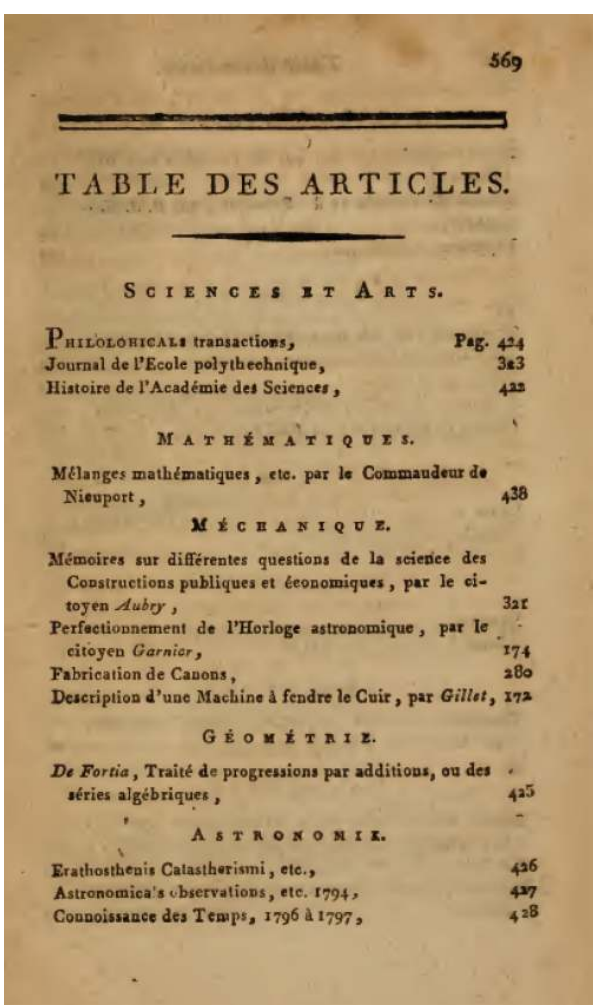

5 Afin de déterminer la cohérence des différents domaines de savoir et pour observer les logiques de voisinage entre ces différents territoires savants, nous étudierons la récurrence de certaines associations de rubriques dans les tables du Magasi ${ }^{16}$. Nous parlons de "territoires" savants et non de disciplines parce que les limites entre plusieurs domaines sont toujours incertaines et souvent mobiles. La structure de l'Institut national entre 1795 et 1803 sert de point de référence. Il est alors partagé en trois classes : les sciences physiques et mathématiques (classe 1); les sciences morales et politiques (classe 2) ; la littérature et les Beaux-Arts (classe 3). 
Fig. 2 : Modèle simplifié de la distribution en rubriques des tables du Magasin encyclopédique. 1795-1816

MAGASIN ENCYCLOPÉDIQUE: MODÈLE DE TABLE DES MATIÈRES (nb. art. > 10)

\begin{tabular}{|c|c|c|}
\hline$=$ CLASSE 1 & $=$ CLASSE 2 & $=$ CLASSE 3 \\
\hline Mathématiques (1a-3) & Economie (1a-1) & Grammaire (1a-1) \\
\hline Astronomie (1a-1) & Morale (1a-2) & Ecriture (4a-6) \\
\hline Mécanique (1a-6) & Politique (2a-2) & Philologie (3a-6) \\
\hline Physique (1a-3) & Législation (2a-2) & Langues (6a-2) \\
\hline Météorologie (1a-1) & Géographie (1a-1) & Littérature (1a-1) \\
\hline Chimie (1a-3) & Histoire (1a-1) & Poésie (1a-1) \\
\hline Histoire naturelle (1a-1) & Antiquités (1a-1) & Romans (1a-4) \\
\hline Anatomie (1a-1) & & Peinture $(8 a-6)$ \\
\hline Médecine (1a-1) & & Sculpture (1806-4) \\
\hline \multirow[t]{5}{*}{ Agriculture (1a-3) } & & Architecture (1a-3) \\
\hline & & Musique (2a-5) \\
\hline & Actualités savantes (1a-1) & Théâtre (1a-1) \\
\hline & Beaux-Arts (1a-2) & \\
\hline & Artttechnologie (1a-1) & \\
\hline
\end{tabular}

Les indications de volumes indiquent la première apparition de la rubrique dans le périodique.

Fig. 3 : Schéma d'organisation de l'Institut national en classes et sections. 1795-1803.

INSTITUT NATIONAL: ORGANISATION DES SECTIONS

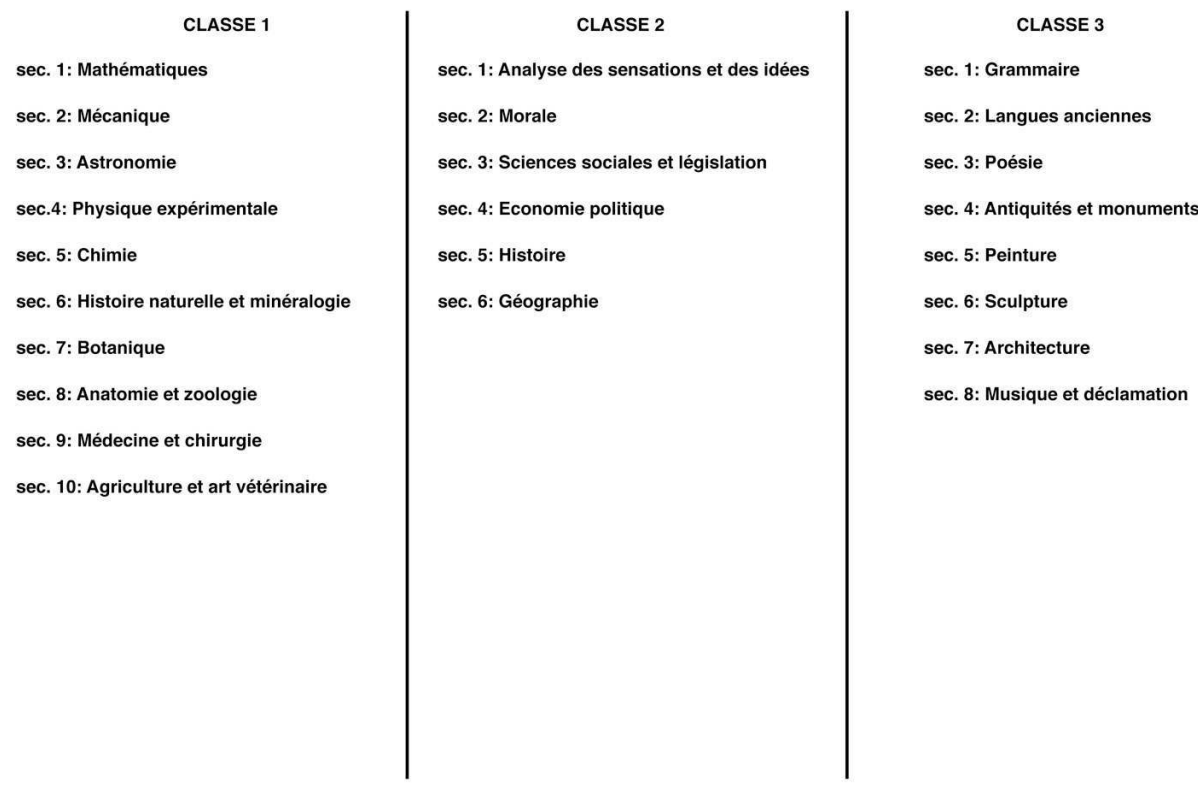

6 La distribution des tables des matières du Magasin encyclopédique reprend, pour l'essentiel, le découpage de l'Institut National en classes et sections. On observe néanmoins quelques petites différences. Ainsi quelques rubriques régulières du périodique n'existent pas sous 
forme de sections à l'Institut national, par exemple la Météorologie, l'Écriture ou le Roman. À l'inverse, aucune rubrique du Magasin ne correspond à la section Analyse des sensations et des idées de l'Institut, associée au courant des Idéologues. Il nous semble bien que l'encyclopédisme du moment révolutionnaire ne saurait se réduire à ce seul mouvement intellectuel. La principale différence entre ces deux lieux de l'encyclopédisme tient cependant à la position des Antiquités : dans le périodique, elles sont dans le voisinage de l'histoire; à l'Institut national, elles sont proches des langues anciennes, de la poésie et des Beaux-Arts. Ce choix s'explique par le refus de Millin d'associer l'archéologie et «l'antiquité figurée » à l'art du dessin comme il l'écrit dans une lettre à Karl August Böttinger ${ }^{17}$. Malgré ces petites différences, il est incontestable que la structure de l'Institut national ait pu servir de modèle à l'ordonnancement des tables du périodique de Millin, d'autant que la disposition de la table du premier Magasin, celui de 1792/1793, était très différente. Dans les vingt années d'existence de la revue, on n'observe aucun changement significatif dans la distribution générale des tables, même dans les mois qui suivent la refonte de l'Institut national marquée par la suppression de la deuxième classe et le dédoublement de la troisième le 3 brumaire an X (24 janvier 1803) ${ }^{18}$. En fait, ce n'est que momentanément, vers 1810, que l'on observe du désordre dans les tables du Magasin.

\section{Les territoires savants}

7 Certains domaines savants voient généralement se succéder les mêmes rubriques. C'est d'abord le cas des savoirs qui relèvent de la troisième classe de l'Institut national pour lesquels chaque rubrique désigne un genre littéraire ou un support artistique. Pour la première et deuxième classe, on repère également quelques domaines cohérents.

- Le territoire naturaliste réunit les rubriques Histoire naturelle, Minéralogie, Botanique, et Zoologie, celle-ci étant elle-même fragmentée en une multitude de sous-rubriques, de l'entomologie à la cétologie en passant par la batractologie (sic). De manière moins systématique, on retrouve souvent dans les parages immédiats de l'histoire naturelle les rubriques Pétrifications, Physiologie, Anatomie comparée ou Géologie.

- Le domaine antiquaire rapproche les rubriques Antiquités, Archéologie, Archéographie, Numismatique, Glyptique, Paléographie etc ${ }^{19}$.

- Le champ médical rassemble les rubriques Médecine, Chirurgie, Encéphalo-craniologie, Pharmacie, Vaccine, Galvanisme et le plus souvent, Anatomie et Physiologie.

- Le domaine géographique dispose près de la rubrique Géographie, celles des Voyages, de la Statistique et la Topographie.

8 Certains domaines de savoir sont beaucoup moins structurés à l'image de l'Économie qui regroupe des rubriques nombreuses et variées, de la Philanthropie à l'Économie politique en passant par les Finances, les Encouragements ou l'Impôt. Entre ces différents territoires savants, qui correspondent pour l'essentiel au découpage en sections de l'Institut national, quelques rubriques font régulièrement office de transition comme l'Anatomie souvent disposée entre l'histoire naturelle et la médecine ou bien encore l'économie rurale et la technologie à cheval entre le domaine scientifique et celui de l'économie. À la différence des territoires savants en voie de constitution disciplinaire, quelques rubriques voguent d'un domaine à l'autre, au gré des volumes du Magasin. Parmi ces savoirs baladeurs, on relève quelques $\operatorname{cas}^{20}$. 
- La Cosmographie et la Cosmologie sont généralement à proximité de l'Astronomie (4 cas) et exceptionnellement de la Géographie $(1 \mathrm{cas})^{21}$.

- La Navigation est tantôt proche de l'Astronomie et des Arts militaires (5 cas), tantôt du domaine économique (4 cas) ou, exceptionnellement, de la Géographie (1 cas) ${ }^{22}$.

- La Géologie, presque toujours disposée à proximité immédiate de l'Histoire naturelle (22 cas), est parfois placée à côté de la Physique (4 cas) ou en position de transition entre les deux domaines $(2 \text { cas })^{23}$.

- La Technologie est dans une position très incertaine. Très souvent, la rubrique est à proximité de celle d'Agriculture, autre art réputé utile (18 cas). Parfois, elle est insérée dans le domaine du commerce auquel elle pourrait offrir de nouveaux produits (10 cas). Plus rarement, elle est placée à proximité des Beaux-Arts, ce qui est une manière d'associer ces derniers aux Arts mécaniques $(5$ cas). Dans de très nombreux cas, la rubrique est cependant dans une position incertaine ou de transition (26 cas) ${ }^{24}$.

- La Psychologie est partagée entre les sciences morales ( 2 cas) et les savoirs médicaux (1 (as) ${ }^{25}$.

- L'Anthropologie est placée soit au sein du domaine naturaliste (2 cas), soit parmi les sciences médicales ( 4 cas), soit parmi les sciences morales $(1 \mathrm{cas})^{26}$. Ainsi, la rubrique Anthropologie traite de l'homme tantôt comme être moral tantôt comme espèce animale.

De cette liste, délibérément un peu longue, on peut tirer trois observations plus générales. Les rubriques qui migrent le plus régulièrement d'un domaine savant à un autre sont celles qui sont placées à cheval entre les sciences physiques et mathématiques et les sciences morales et politiques, telles que ces deux ensembles sont définis par la structure de l'Institut national. Au contraire, la position des rubriques que l'on peut rattacher au domaine littéraire est artistique est très stable pendant les vingt années de publication du Magasin. En outre, on observe qu'à l'exception remarquable de la rubrique Anthropologie, ces pérégrinations ne révèlent pas d'un partage intérieur au sein de chacun des territoires savants. Ainsi, des articles aux sujets très proches et présentés sous une même rubrique peuvent être disposés à tel endroit dans un volume et à tel autre dans le suivant. Enfin, nous n'observons pas de claire évolution de la position de chacune de ces rubriques au sein de la table des matières. Ces sciences sont baladeuses; elles se promènent d'un lieu à un autre de la table; elles ne sont pas en transit à la recherche d'une place définitive.

\section{Le poids des savoirs}

Dans l'avertissement du premier volume de la deuxième année, Millin précise le contenu des futurs numéros du Magasin : « un tiers sera destiné aux sciences physiques, et les deux autres tiers à l'histoire, à la littérature et aux arts. La partie des spectacles qui avait été abandonnée y sera reprise; on y trouvera également des notices sur les savants et les hommes de lettres de tous les pays dont on aura à regretter la perte; enfin, les nouvelles littéraires mettront au courant de ce qui se passe en ce genre en Europe et des nouveautés qu'on y publie $»^{27}$. Ainsi, dès 1796 , le directeur du périodique annonce une répartition équilibrée des articles entre trois "parties» qui correspondent aux trois classes de l'Institut. L'objet de l'analyse statistique est dès lors de confronter le projet énoncé par Millin à la distribution effective des rubriques au sein du Magasin. On propose de distribuer les articles du Magasin au sein de la nomenclature des classes et des sections de l'Institut national dans sa structure de 1795 . On prend l'article comme unité 
statistique, malgré la relative grossièreté de cette norme de mesure. Pour la répartition par classe, on dispose pour les 122 volumes d'environ 9.700 articles, soit environ 80 par volume, sans compter les articles indiqués dans les rubriques généralistes ou inclassables, ou rangés dans les actualités littéraires ou les annonces de spectacles ${ }^{28}$. On a retenu le premier volume de l'année 1803 pour partager en deux périodes la série du Magasin, ce volume faisant immédiatement suite à la réforme de l'Institut.

Fig. 4 : Répartition des articles du Magasin encyclopédique selon le découpage en sections de l'Institut national.

1795-1802

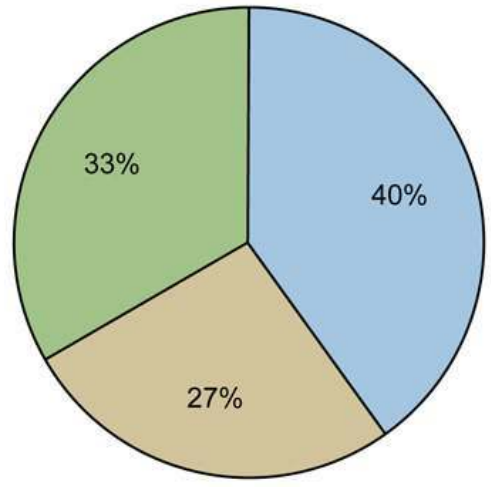

$1803-1816$

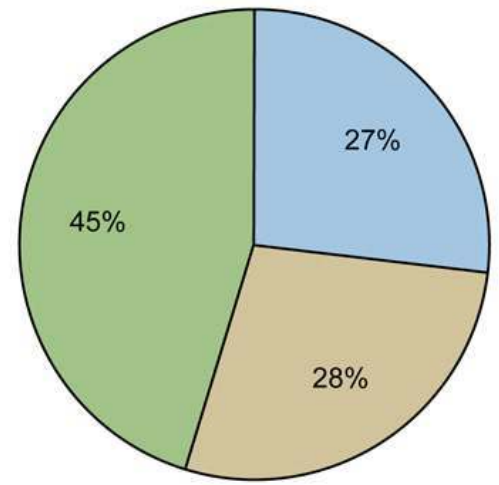

Classe $1 \bigcirc$ Classe $2 \bigcirc$ Classe 3 équilibrée des articles entre les trois classes de l'Institut national. Pour la classe des sciences morales et politiques (cl. 2), la stabilité est remarquable entre les deux périodes, et ce malgré la suppression de la classe en 1803, principale conséquence de la réforme. Pour la classe des sciences (cl. 1) et celle de littérature (cl. 3), on observe entre les deux périodes un renversement de leur poids relatif : entre 1795 et 1802, les sciences ont la plus belle place; celle-ci revient aux lettres et aux arts après 1803. Le programme de Millin d'une répartition harmonieuse entre les trois vastes ensembles des connaissances humaines semble à peu près réalisé. L'essentiel est pourtant bien le maintien de la place des sciences morales et politiques après 1803 , ce qui peut être lu comme une forme de résistance à l'offensive napoléonienne contre les Idéologues ${ }^{29}$.

Pour la répartition par section, on dispose pour les 122 volumes d'un peu moins de 7500 articles. Cela représente un cinquième d'articles en moins que pour l'analyse de la répartition par classe, certaines rubriques n'ayant pu être situées dans le découpage en sections de l'Institut. On peut présenter les différentes classes en commençant par la première, celle des Sciences physiques et mathématiques. 
Fig. 5 : Répartition des articles de Sciences physiques et mathématiques du Magasin encyclopédique selon le découpage en sections de l'Institut national.

1795-1802

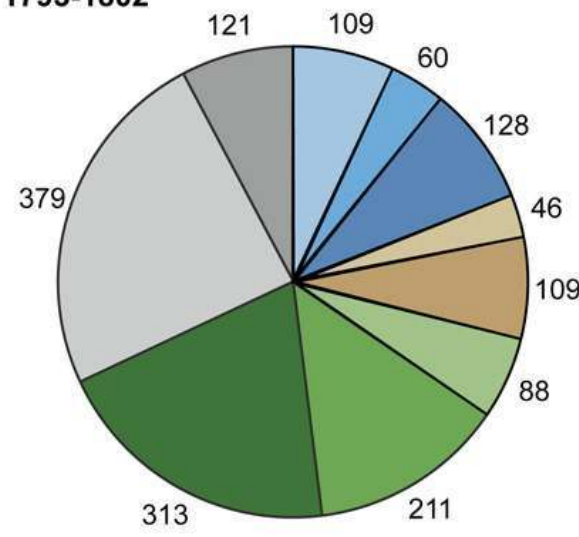

\section{3-1816}

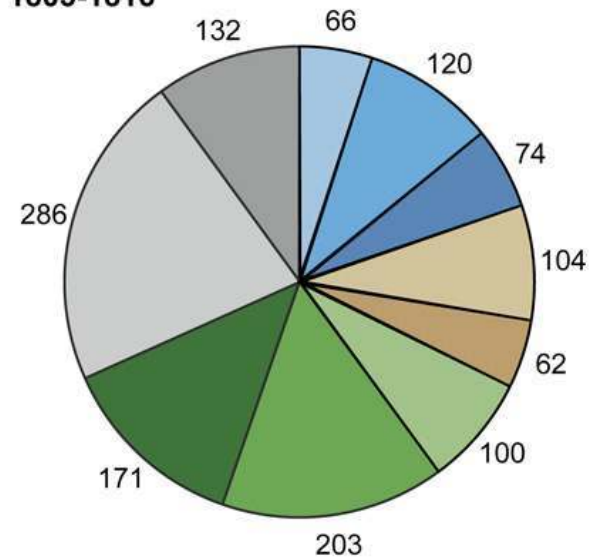

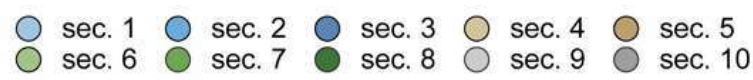

Remarque: sec. 1, Mathématiques; sec. 2, Mécanique; sec. 3, Astronomie ; sec. 4, Physique expérimentale ; sec. 5, Chimie ; sec. 6, Histoire naturelle et minéralogie ; sec. 7, Botanique ; sec. 8, Anatomie et zoologie; sec. 9, Médecine et chirurgie; sec. 10, Agriculture et art vétérinaire.

Au travers d'environ 2900 articles, les dix sections de la classe des sciences sont représentées dans les rubriques du Magasin encyclopédique. Trois ensembles sont nettement sur-représentés et rassemblent plus de la moitié de l'ensemble des articles : la botanique; l'anatomie et la zoologie ; la médecine et la chirurgie. Durant les vingt années de publication du Magasin, particulièrement entre 1795 et 1802, on voit s'affirmer le primat des sciences d'observation et, plus encore, des savoirs médicaux, sur les sciences physico-mathématiques. L'importance de l'histoire naturelle s'explique aussi par le parcours personnel de Millin, membre fondateur de la Société d'histoire naturelle de Paris en $1790^{30}$.

Pour la deuxième classe, celle des sciences morales et politiques, la répartition est la suivante ${ }^{31}$. 
Fig. 6 : Répartition des articles de Sciences morales et politiques du Magasin encyclopédique selon le découpage en sections de l'Institut national

1795-1802

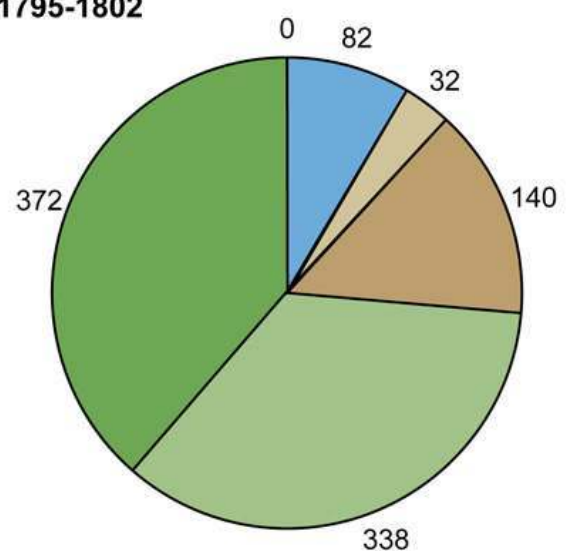

1803-1816

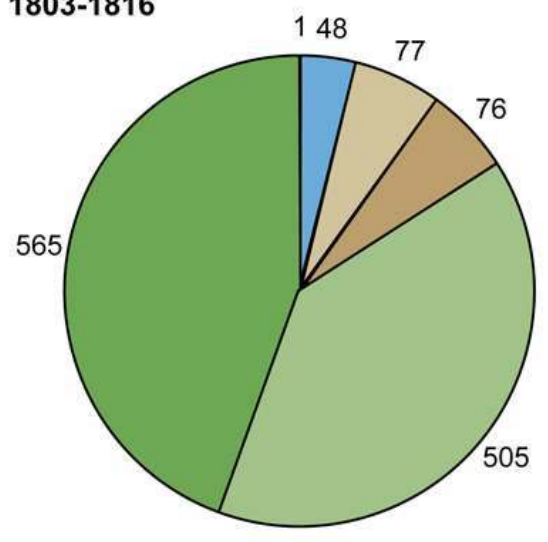

$\bigcirc$ sec. $1 \bigcirc \sec .2 \bigcirc$ sec. $3 \bigcirc$ sec. $4 \bigcirc$ sec. $5 \bigcirc$ sec. 6

Remarque : sec. 1, Analyse des sensations et des idées ; sec. 2, Morale; sec. 3, Sciences sociales et législation; sec. 4, Économie politique; sec. 5, Histoire; sec. 6, Géographie.

À l'exception de l'Analyse des sensations et des Idées, toutes les sections de la classe des sciences morales et politiques sont représentées dans plus de 2.200 articles du Magasin encyclopédique. Les rubriques correspondant aux deux sections Histoire et Géographie de l'Institut national ont la plus belle part, rassemblant environ $80 \%$ des articles. Entre les deux périodes, la part relative de ces deux domaines tend d'ailleurs à croître au détriment des quatre premières sections davantage liées au mouvement des Idéologues. En valeur absolue, les articles de Morale, Sciences sociales et législation et d'Économie politique se font plus rares après 1803. Il semble que contrairement à la Décade, le Magasin encyclopédique accorde une place très réduite à l'Idéologie. En tant que telle, celle-ci ne fait d'ailleurs l'objet que d'un seul article publié tardivement en $1805^{32}$. Pendant ces vingt années, il nous semble que l'encyclopédisme n'est pas seulement porté par les Idéologues. Millin en propose une autre version qu'il décrit comme « purement savante » et très peu politique.

Pour la troisième classe, celle de Littérature et Beaux-Arts, les articles se répartissent comme suit ${ }^{33}$. 
Fig. 7 : Répartition des articles de Littérature et Beaux-Arts du Magasin encyclopédique selon le découpage en sections de l'Institut national

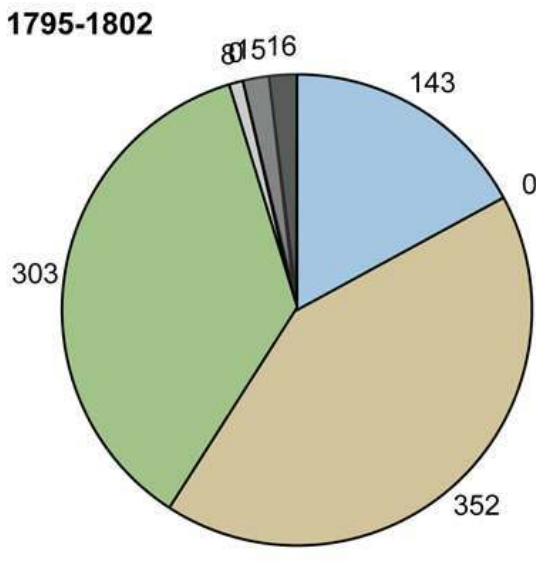

sec.

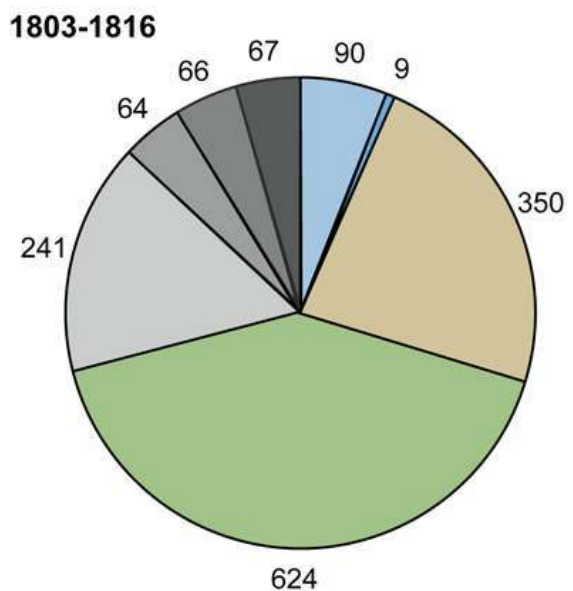

sec. $5 \bigcirc \sec .6$

sec. 7

Remarque : sec. 1, Grammaire ; sec. 2, Langues anciennes ; sec. 3, Poésie ; sec. 4, Antiquités et monuments ; 5 , Peinture ; 6, Sculpture ; 7, Architecture ; 8, Musique et déclamation.

Entre 1795 et 1802, trois groupes de rubriques renferment la quasi-totalité des articles de Littérature et Beaux-Arts publiés dans le Magasin. Elles correspondent aux sections Grammaire, Antiquités et monuments et Poésie de la troisième classe de l'Institut. Entre 1803 et 1816, trois domaines renferment encore la majeure partie des articles. Ils correspondent aux sections Peinture, Poésie et Antiquités et monuments. L'affirmation continue des antiquités est à mettre en relation avec l'intérêt toujours plus marqué de Millin figpour les antiques à partir de 1800 et notamment de son voyage dans les départements du Midi de la France. On peut voir dans la place plus importante accordée aux arts du dessin un écho de leur position privilégiée dans la France impériale.

Entre 1795 et 1816, le Magasin est un des hauts lieux de l'encyclopédisme en France et peut-être en Europe. Sa publication tient d'abord à un homme, Millin, qui, sous la Restauration, tente encore de faire revivre sa revue savante sous le nouveau nom d' Annales encyclopédiques. L'expérience lancée en 1817 ne survit pas à la mort de son principal rédacteur l'année suivante. Pendant sa vingtaine d'années d'existence, le Magasin propose à ses lecteurs presque 15.000 articles dont les titres sont ordonnés en rubriques dans les tables des matières des 122 volumes. L'ordonnancement des savoirs que propose Millin dans ces tables est très proche de la distribution en 3 classes et 24 sections qu'a connue l'Institut national entre 1795 et 1803. On note néanmoins quelques différences dont la plus remarquable est la place des Antiquités aux côtés de l'Histoire et non des Arts et de la Littérature antique. Cette organisation des articles se maintient d'ailleurs par-delà la suppression de la deuxième classe en 1803 et après cette date, on trouve encore la Géographie à proximité de l'Histoire, malgré son rapatriement institutionnel dans la première classe de l'Institut. Dans cette affirmation têtue du projet encyclopédique, on peut sans doute voir une forme de résistance à "l'organisation impériale des savoirs ». Mais l'encyclopédisme qui s'affirme dans le périodique ne saurait être assimilé au courant des Idéologues qui domine la première période de l'Institut. Millin défend une conception délibérément apolitique de la réunion des sciences et des 
arts, peut-être une version plus sage et moins articulée de l'encyclopédisme. L'étude statistique des articles rangés en rubriques révèle un relatif équilibre entre les trois domaines des connaissances humaines, les Sciences physiques et mathématiques, les Sciences morales et politiques, la Littérature et les Beaux-Arts. Pourtant, il apparaît que la part des sciences baisse tendanciellement tout au long de la période : $39 \%$ des articles entre 1795 et $1799 ; 37 \%$ entre 1800 et $1805 ; 24 \%$ entre 1806 et $1810 ; 20 \%$ entre 1811 et 1816. A contrario, la place de la Littérature et des Beaux-arts, auxquels on a joint les " Antiquités ", augmente brutalement au début du nouveau siècle : $29 \%$ des articles entre 1795 et $1799 ; 25 \%$ entre 1800 et $1805 ; 38 \%$ entre 1806 et $1810 ; 37 \%$ entre 1811 et 1816 . Il est difficile d'interpréter ces mouvements en leur donnant une cause unique. Ce chassécroisé peut se lire comme la traduction de l'intérêt toujours plus marqué de Millin pour les antiquités au détriment de l'histoire naturelle qui occupe une place beaucoup plus restreinte dans le récit de son Voyage dans les départemens du midi de la France ${ }^{34}$. Millin est toujours plus antiquaire et de moins en moins botaniste. Mais ce renversement peut aussi s'expliquer par l'évolution du marché de la presse. De prime abord, on est surpris par la raréfaction des articles d'anatomie comparée après 1803, au moment où Cuvier devient progressivement le personnage central des sciences naturelles. Mais en 1802, le Muséum lance ses Annales qui se substituent rapidement à tout un ensemble de périodiques encyclopédiques où étaient jusqu'alors publiés les articles d'histoire naturelle. Immédiatement, l'habile Cuvier y fait passer tous ses travaux savants ${ }^{35}$. Pour aller plus loin, il faudrait confronter l'agencement des connaissances humaines dans le Magasin avec celui proposé dans d'autres lieux encyclopédiques. On pense à d'autres titres de la presse périodique, à commencer par la Décade philosophique ainsi qu'aux grands dictionnaires et encyclopédies, et d'abord à la Méthodique ${ }^{36}$. On pense aussi aux réunions de savants, que ce soit la Commission temporaire des arts ou les sociétés savantes de Paris et de province. On pense enfin aux circulations d'objets entre les collections parisiennes, thème que nous développerons ailleurs.

\title{
ANNEXES
}

\section{Liste des numéros du Magasin encyclopédique de 1795 à 1804 compris}

\author{
1a-1 : Année 1, tome I, (an III/1795). \\ 1a-2 : Année 1, tome II, (an III/1795). \\ 1a-3 : Année 1, tome III, (an III/1795). \\ 1a-4 : Année 1, tome IV, (an IV/1795). \\ 1a-5 : Année 1, tome V, (an IV/1795). \\ 1a-6 : Année 1, tome VI, (an IV/1795).
}


2a-1 : Année 2, tome I, (an IV/1796).

2a-2 : Année 2, tome II, (an IV/1796).

2a-3 : Année 2, tome III, (an IV/1796).

2a-3 : Année 2, tome III, (an IV/1796).

2a-4 : Année 2, tome IV, (an V/1796).

2a-5 : Année 2, tome $V$, (an V/1797).

2a-6 : Année 2, tome VI, (an V/1797).

3a-1 : Année 3, tome I, (an V/1797).

3a-2 : Année 3, tome II, (an V/1797).

3a-3 : Année 3, tome III, (an V/1797).

3a-4 : Année 3, tome IV, (an VI/1797).

3a-5 : Année 3, tome IV, (an VI/1797).

3a-6 : Année 3, tome VI, (an VI/1798).

4a-1 : Année 4, tome I (an VI/1798).

4a-2 : Année 4, tome II (an VI/1798).

4a-3 : Année 4, tome III (an VII/1798).

4a-4 : Année 4, tome IV (an VII/1798).

4a-5 : Année 4, tome V (an VII/1799).

4a-6 : Année 4, tome VI (an VII/1799).

5a-1 : Année 5, tome I (an VII/1799).

5a-2 : Année 5, tome II (an VII/1799).

5a-3 : Année 5, tome III (an VII/1799).

5a-4 : Année 5, tome IV (an VIII/1799).

5a-5 : Année 5, tome V (an VIII/1799).

5a-6 : Année 5, tome VI (an VIII/1799).

6a-1 : Année 6, tome I (an VIII/1800).

6a-2 : Année 6, tome II (an VIII/1800).

6a-3 : Année 6, tome III (an VIII/1800).

6a-4 : Année 6, tome IV (an VIII/1800).

6a-5 : Année 6, tome V (an IX/1801).

6a-6 : Année 6, tome VI (an IX/1801).

7a-1 : Année 7, tome I (an IX/1801).

7a-2 : Année 7, tome II (an IX/1801).

7a-3 : Année 7, tome III (an IX/1801).

7a-4 : Année 7, tome IV (an IX/1801).

7a-5 : Année 7, tome V (an IX/1801).

7a-6: Année 7, tome VI (an IX/1801).

8a-1 : Année 8, tome I (an X/1802).

8a-2 : Année 8, tome II (an X/1802).

8a-3 : Année 8, tome III (an XI/1802).

8a-4 : Année 8, tome IV (an XI/1802).

8a-5 : Année 8, tome V (an XI/1803).

8a-6 : Année 8, tome VI (an XI/1803).

9a-1 : Année 9, tome I (an XI/1803).

9a-2 : Année 9, tome II (an XI/1803). 
9a-3 : Année 9, tome III (an XI/1803).

9a-4 : Année 9, tome IV (an XI/1803).

9a-5 : Année 9, tome V (an XII/1804).

9a-6 : Année 9, tome VI (an XII/1804).

À partir de 1805, la tomaison du Magasin encyclopédique fait simplement suivre l'année et le numéro du volume.

\section{NOTES}

1. Sur Millin, voir notamment: Charles-Guillaume KRAFFT, « Notice sur Aubin-Louis Millin», Annales encyclopédiques, vol. 6 (1818), p. 5-84.

2. Voir l'article très érudit de: Alain RuIz, «Autour du Magasin encyclopédique. Les amis et visiteurs germaniques d'Aubin-Louis Millin à Paris, de Thermidor à la Restauration ", dans Geneviève ESPAGNE et Bénédicte SAVOY, (dir.), Aubin-Louis Millin et l'Allemagne. Le Magasin encyclopédique - Les lettres à Karl August Böttiger, Hildesheim, Zürich, New York, Georg Olms, Europea memoria, 2005, p.5-57. On sait aussi que des premiers thés mondains sont organisés chez Warens en 1795. On y discute, entre autres choses, de la rédaction du journal et on y rencontre «tout ce que Paris possède en savants et beaux esprits, en naturalistes, chimistes, spécialistes de philologie latine et grecque, orientalistes, poètes lyriques, tragiques, auteurs d'opéras-comiques, numismates, antiquaires, historiens, géographes, etc. ».

3. Parmi les habitués, on rencontre: Antoine François Fourcroy, Jean-Fréderic Hermann, Wilhelm von Humboldt, Alexandre von Humboldt, Thomas Bugge, Friedrich Johann Lorenz Meyer, Charles de Villers, Friedrich Schlegel ou Georges Cuvier. Dans une lettre à Brinkmann, Wilhelm von Humboldt écrit que « toute la langue allemande était réunie là ».

4. Alain RuIZ, « Autour du Magasin encyclopédique... », art. cit., p. 35.

5. Geneviève ESPAGNE et Bénédicte SAVOY, (dir.), Aubin-Louis Millin et l'Allemagne..., op. cit.

6. Cité dans : Cécilia HURLEY, «Un cosmopolitisme journalistique : les généalogies du Magasin encyclopédique", dans Geneviève ESPAGNE et Bénédicte SAVoY, (dir.), Aubin-Louis Millin et l'Allemagne..., op. cit., p. 113.

7. Magasin encyclopédique, $2^{\text {ème }}$ année, vol. 1 (1796), p. ix, avertissement : « c'est moi qui ai conçu le projet de ce Journal, et qui, depuis son origine, ai dirigé seul son exécution». En 1816, le gouvernement soumet la presse périodique à un impôt. François-Joseph Noël: ancien clerc, régent de collège puis membre du Comité d'Instruction publique et publiciste ; Israël Warens : homme d'affaire hambourgeois, premier bailleur de fonds.

8. Par exemple, sur le Journal des mines, voir : Isabelle LABOULAIS, « Entre minéralogie et statistique territoriale : les enquêtes du Journal des mines entre l'an III et l'an VII », Revue d'histoire moderne et contemporaine, vol. 55, $\mathrm{n}^{\circ}$ 4, octobre-décembre 2008, p. 57-81, notamment p. 58.

9. Eugène HATTIN, Bibliographie historique et critique de la presse périodique française, Paris, Firmin Didot Frères, fils et Cie, 1866, p. 312-313 ; RÉGALDO, Un milieu intellectuel. La Décade philosophique, 1794-1807, Lille et Paris, Service de reproduction des thèses et Honoré Champion, 1976, t. I, p. 199-202 et t. V, p. 948-952.

10. John PINKERTON, Recollections of Paris in the Years 1802-1805, London, Longman, Hurst Rees et Orme, 1806, tome 2, p. 303: «I have been well informed that of the Magazin (sic) Encyclopédique, and the Decade, now Revue, the sales rarely passes three hundred ».

11. Geneviève ESPAGNE «Introduction », dans Geneviève ESPAGNE et Bénédicte SAVoy, (dir.), AubinLouis Millin et l'Allemagne..., op. cit., p. xii, n. 16. L'auteur observe que l'année de référence, 1803, est pour les milieux «philosophes » dont le Magasin est proche une année de repli après la phase d'expansion entre 1795 et 1800. 
12. Minerva, 1795, vol. 3, p. 196-197 ; cité dans : Alain RUIZ, « Autour du Magasin encyclopédique... », art. cit., p. 12.

13. Magasin encyclopédique, $2^{\text {ème }}$ année, vol. 1 (1796), p. vii-ix.

14. Décade philosophique, vol. 3 (septembre à novembre 1794), p. 471.

15. Pascale RABAULT, «Réseaux internationaux de l'orientalisme naissant. Le Magasin encyclopédique comme relais du savoir sur l'Orient ", dans Geneviève ESPAGNE et Bénédicte SAVoY, (dir.), Aubin-Louis Millin et l'Allemagne..., op. cit., p. 178-179; Gérard LAUDIN, « Les comptes rendus d'ouvrages historiques allemands dans le magasin encyclopédique ", dans Geneviève ESPAGNE et Bénédicte SAvoy, (dir.), Aubin-Louis Millin et l'Allemagne..., op. cit., p. 191-192 ; Pascale HUMMEL, «Le passé présent : philologie 'périodique' dans le Magasin encyclopédique ", dans Geneviève ESPAGNE et Bénédicte SAvoy, (dir.), Aubin-Louis Millin et l'Allemagne..., op. cit., p. 151-160.

16. On peut modéliser ces logiques en supprimant les rubriques qui sur la totalité des volumes comprennent moins de 10 articles et en laissant de côté les situations exceptionnelles.

17. Cité dans Bénédicte SAVoY, "Savoir archéologique partagé. Les lettres d'Aubin-Louis Millin à Karl August Böttiger. 1797-1817 », dans Geneviève ESPAGNE et Bénédicte SAVoY, (dir.), Aubin-Louis Millin et l'Allemagne..., op. cit., p. 76 : «vous croiriez avec peine qu'on ignore presqu'ici ce qu'est l'archéologie et l'antiquité figurée, on croit que le seul intérêt des monumens est de donner des règles de Dessin et de belles proportions ".

18. Le Magasin encyclopédique en fait mention dans son volume 8a-5, premier volume de l'année 1803.

19. Pour Millin, les mots « antiquité » et " archéologie », presque synonymes, recouvrent toutes les formes de la connaissance de l'antiquité. Voir : Marie-Renée DIOT-DURIATTI, «Jérémie Jacques Oberlin correspondant d'Aubin-Louis Millin », dans Geneviève ESPAGNE et Bénédicte SAVoY, (dir.), Aubin-Louis Millin et l'Allemagne..., op. cit., p. 105 : «archaelogie, nom composé de deux mots grecs, et qui signifie connaissance des Antiquités, comme zoologie signifie connaissance des animaux, ichtyologie connaissance des poissons etc. ». Voir aussi : Pascale HUMMEL, «Le passé présent... ", art. cit., p. 149 : l'archéologie est « la science connue ordinairement sous le nom d'Antiquités».

20. Pour simplifier la lecture, on indique seulement les repères des volumes. Ainsi $9 a-3$ doit se lire $9^{\text {ème }}$ année, vol. 3 et 1812-3, Année 1812, vol. 3. Pour la tomaison des volumes publiés entre 1795 et 1804 , on se réfèrera à la liste présentée en annexe.

21. Cosmographie et Cosmologie : voisin de l'Astronomie (3a-2, 4a-1, 4a-6, 1816-2); proche de la Géographie (9a-2).

22. Navigation : proche de l'Astronomie et l'Art militaire (1a-1, 1a-3, 1a-4, 1806-5, 1812-1), de la Géographie (7a-5, 8a-5 , domaine de l'économie et du commerce (8a-6, 1805-1, 1805-3, 1810-3) ou de la technologie à propos de la boussole (1809-6).

23. Géologie : dans Histoire naturelle (1a-1, 3a-2, 3a-5, 3a-6,6a-2, 6a-3, 7a-3, 8a-3, 9a-1, 1806-1, 1806-3, 1807-2, 1808-3, 1808-5, 1809-3, 1809-4, 1809-5, 1810-6, 1811-3, 1812-3, 1812-4, 1813-4 avant ou après Minéralogie si la rubrique existe sinon dans Histoire naturelle); proche de la Physique (4a-5, 7a-2, 7a-6, 9a-2); situation de transition (8a-5, 1809-2).

24. Technologie : banlieue de l'Agriculture pour ces fabrications et méthodes (3a-1, 4a-3, 4a-5, 4a-6, 5a-1, 5a-5, 6a-2, 7a-2, 7a-4, 9a-1, 9a-5, 1806-1, 1806-2, 1806-3, 1806-4, 1808-1, 1808-2, 1811-5) ; près du Commerce (6a-1, 7a-5, 8a-1, 8a-6, 9a-3, 1805-1, 1805-2, 1805-3, 1807-5, 1812-1, ) ; après les Beaux-arts pour des arts et manufactures (5a-6, 9a-2, 1806-5, 1806-6, 1807-4) ; position incertaine ou de transition (7a-6, 9a-4, 9a-6, 1805-4, 1805-5, 1805-6, 1807-2, 1807-6, 1808-4, 1808-6, 1809-2, $1809-3,1810-2$, 1810-3, 1811-1, 1811-3, 1811-4, 1812-2, 1812-3, 1812-6, 1813-1, 1813-5, 1813-6, 1815-5, 1815-6, 1816-2).

25. Psychologie: dans le domaine des sciences morales (1809-4, entre Éducation et Morale; 1809-5, après Morale); au sein des sciences médicales (1813-5, après Chirurgie). 
26. Anthropologie : dans l'Histoire naturelle avec un article sur les variétés humaines d'après Homère et un autre de Blumenbach (1a-4, entre la Botanique et l'Entomologie ; 1805-5, avant l'Entomologie), parmi les sciences morales avec deux articles qui traitent de l'Homme comme animal moral (8a-4, entre la rubrique Morale et la rubrique Éducation), dans le domaine des sciences médicales avec, notamment, des articles sur l'hygiène, la physiognomonie de Gall (9a-2, entre Physiologie et Médecine ; 1805-5, entre Physiologie et Hygiène ; 1806-1 et 1806-2 : avant Médecine).

27. Magasin encyclopédique, $2^{\text {ème }}$ année, vol. 1 (1796), p. vii-ix.

28. Si l'on tient compte de ces catégories - à l'exception de celle d'«histoire littéraire » - on obtient, d'après nos calculs, 12.310 articles dans les tables des 122 volumes du Magasin, soit environ 92 articles par volume entre 1795 et 1799, 126 entre 1800 et 1805, 98 entre 1806-1810, et 65 entre 1811 et 1816. Cela nous semble assez bien indiquer le degré d'activité du Magasin, fort au début du nouveau siècle et en net déclin à la fin de l'Empire.

29. Sur cette question, voir: Jean-Luc CHAPPEY, «Héritages républicains et résistances à 'l'organisation impériale des savoirs' ", Annales historiques de la Révolution française, $\mathrm{n}^{\circ} 346,2006$, p. 97-120.

30. La rubrique « histoire naturelle » que nous n'avons pas retenue contient beaucoup d'autres articles (130 au total soit 61 pour la période 1795-1802 et 69 pour la période 1803-1816).

31. Nous avons intégré la catégorie « voyages » dans le recensement des articles de géographie, les sujets sur le voyage occupant une part importante de la section Géographie de l'Institut, comme l'a montré Hélène Blais: Hélène BLAIS, «La Géographie académique entre sciences et Belles-Lettres (autour de la scission de 1803)», dans Hélène BLAIS et Isabelle LABouLAIS, Géographies plurielles. Les sciences géographiques au moment de l'émergence des sciences humaines (1750-1850), Paris, L'Harmattan, 2006, p. 95-112.

32. Magasin encyclopédique, année 1805, vol. 1.

33. Nous avons rangé les "Antiquités » dans la troisième classe pour respecter le schéma d'organisation de l'Institut dans la construction de nos statistiques bien que le Magasin les place dans la banlieue de l'histoire comme nous l'avons vu.

34. Aubin-Louis millin, Voyage dans les départemens du midi de la France, Paris, Imprimerie impériale, 1807-1811, 4 tomes en 5 volumes.

35. D'après la liste bibliographique d'Henri DAUDIN, Cuvier et Lamarck. Les classes zoologiques et l'idée de série animale. 1790-1830, Paris, Edition des archives contemporaines, 1983 [1926-1927], vol. 2, p. 285-293. Entre 1795 et 1801, Cuvier publie dans la Décade philosophique (1795), dans le Magasin encyclopédique (1795-1796), dans le Journal de physique (1798-1801) et dans le Bulletin de la Société philomatique (1795-1801). Entre 1802 et 1812, il publie exclusivement dans les Annales du Muséum. Voir aussi: Dorinda oUTRAM, Georges Cuvier. Vocation, Science, and Authority in Post-Revolutionary France, Manchester, Manchester University Press, 1984, p. 67.

36. Cf. Claude BLANCKAERT et Michel PORRET, L'Encyclopédie méthodique (1782-1832). Des Lumières au positivisme, Genève, Droz, 2006.

\section{RÉSUMÉS}

Publié entre 1795 et 1816, le Magasin encyclopédique dirigé par Aubin-Louis Millin rassemble plus de 10.000 articles répartis dans environ 250 rubriques de la table des matières. Le périodique est 
un site idéal pour observer la configuration de l'encyclopédisme entre les débuts du Directoire et la fin de l'Empire. Nous proposons une étude en trois temps. On propose d'abord de modéliser l'enchaînement des savoirs dans les tables du Magasin et de comparer cet ordonnancement avec l'organisation en sections de l'Institut national, son contemporain. On essaye ensuite de déterminer la cohérence et la régularité des différents territoires savants, certains d'entre eux apparaissant mieux engagés que d'autres sur la voie de la constitution disciplinaire des champs de savoir. On présente enfin une analyse statistique du poids relatif de chacun de ces domaines de savoir en distribuant les quelques milliers d'articles du périodique dans des catégories décalquées sur la structure de l'Institut.

INDEX

Mots-clés : Magasin encyclopédique, Aubin-Louis Millin, Institut national, encyclopédisme, savoirs

\section{AUTEUR}

\section{PIERRE-YVES LACOUR}

Université de Montpellier III 